\title{
Homo Sapiens (Human) microRNA 187 Expression Is Dysregulated in Testis of Non-obstructive Azoospermic Men
}

\author{
Fahimeh Piryaei ${ }^{1,2}$, Hossein Mozdarani ${ }^{*}{ }^{*}$, Mohammad Ali Sadighi Gilani ${ }^{3}$, Mansour Ebrahimi ${ }^{4}$ \\ 'Department of Medical Genetics, Faculty of Medical Sciences, Tarbiat Modares University, Tehran, Iran. \\ ${ }^{2}$ Research Center for Molecular Medicine, Hamadan University of Medical Sciences, Hamadan, Iran. \\ ${ }^{3}$ Department of Andrology, Reproductive Biomedicine Research Center, Royan Institute for Reproductive Biomedicine, \\ ACECR, Tehran, Iran. \\ ${ }^{4}$ Department of Biology, School of Basic Sciences, Qom University, Qom, Iran.
}

\section{*Correspondence to Hossein Mozdarani, \\ Email: Mozdarah@modares.ac.ir \\ Received December 30, 2018 Accepted July 13, 2019 \\ Published online June 30, 2020}

\begin{abstract}
Introduction: MiRNA expression alterations are closely related to growth, proliferation, and cell development. In addition, miR-187 has been found to regulate cellular proliferation. Further, spermatogenesis is a highly complicated process which is regulated through several genes targeted by miRNAs. However, no study has completely addressed the etiology of spermatogenic impairments. Accordingly, the present study examined the expression pattern of Homo sapiens (human) microRNA (hsa-mir-187) in the testicular biopsies of infertile men with non-obstructive azoospermia (NOA) and Sertoli cell-only syndrome (SCOS) subtype in comparison with those with obstructive azoospermia (OA).

Methods: Quantitative reverse-transcription polymerase chain reaction analysis was performed to assess the expression level of hsa-mir-187 using the $\Delta \Delta \mathrm{Ct}$ method.

Results: Our data indicated that hsa-mir-187 was significantly up-regulated in the testis of the SCOS group $(P<0.05)$ in comparison with the OA group.

Conclusion: In general, the findings suggest that the differential expression of hsa-mir-187 is linked with male infertility and shed more light on molecular defects that underlie spermatogenic impairments.

Keywords: Non-obstructive azoospermia, Obstructive azoospermia, miR-187, Sertoli cell-only syndrome, Testicular tissue
\end{abstract}

\section{Introduction}

Infertility, as a worldwide reproductive health problem, is estimated to affect nearly $15 \%$ of couples. ${ }^{1}$ Male factor contributes to $50 \%$ of couples' infertility. In $30 \%-40 \%$ of all cases, male infertility occurs in patients without prior infertility conditions and normal findings on physical examination termed "idiopathic male infertility". Azoospermia, which is referred to the lack of sperms in the ejaculate, comprises a remarkable portion of idiopathic male infertility cases and is caused by either primary testicular failure (non-obstructive azoospermia, NOA) or sperm blockage from entering the semen (obstructive azoospermia, OA).

Spermatogenesis as a highly organized, complex process of differentiation is precisely modulated by different posttranscriptional and translational events. ${ }^{3}$ MicroRNAs (18-22 nucleotides) modulate gene expression in different biological processes at the post-transcriptional level through interlocking to the 3' untranslated region (3'UTR) of target mRNAs, which result in the inhibition of translation and/or mRNA degradation. ${ }^{4,5}$ Previous studies obviously elucidated that testicular-expressed miRNAs are involved in the spermatogenesis regulation, and their expression pattern changes as the successive stages of spermatogenesis appear consecutively. To date, several genomewide expression studies have focused on identifying testicular dysregulated miRNAs in infertile men. ${ }^{6-8}$ It is strongly suggested that hsa-miR-187, as a tumor suppressor,

(c) 2020 The Author(s); Published by Zabol University of Medical Sciences. This is an open-access article distributed under the terms of the Creative Commons Attribution License (http://creativecommons.org/licenses/by/4.0), which permits unrestricted use, distribution, and reproduction in any medium, provided the original work is properly cited. 
is critically involved in regulating cellular proliferation. ${ }^{9}$ Nevertheless, the role of miR-187 in male infertility pathogenesis has not been understood yet. Thus, a better understanding of the changes in miRNA expression in patients with impaired spermatogenesis might pave the way to propose new attractive therapeutic targets.

Therefore, the present study aimed to examine the expression pattern of hsa-miR-187-3p in the testis of OA and idiopathic NOA infertile patients. It is hoped that our study increases the understanding of the molecular role of miR-187 in impaired spermatogenesis fertility.

\section{Materials and Methods \\ Participants}

The testicular biopsy specimens of azoospermic individuals referring to the Royan Institute were obtained from patients with idiopathic NOA (Sertoli cell-only syndrome, SCOS, $\mathrm{n}=10$, the mean age $=34.8 \pm 5.20$ years $)$ and patients with OA $(n=10$, the mean age $=36.1 \pm 5.64$ years). Each patient underwent testicular sperm extraction with the goal of diagnostic biopsy for histological examination and/or assisted reproduction. Initially, azoospermia was diagnosed based on showing absent ejaculated spermatozoa in two semen analyze examinations according to the 2010 World Health Organization criteria. $^{10}$ Subsequently, SCOS was empirically defined as having no epididymal and/or testicular spermatozoa. OA was defined as a considerable number of mature spermatozoa sampled by testicular sperm extraction or motile spermatozoa aspirated from microsurgical epididymal sperm aspirations.

Informed consent was obtained from all patients who participated in this study. All included azoospermic men were diagnosed with primary infertility, and patients with recognized medical conditions for their infertility (e.g., the pathologies of the epididymis or vas deferens, undescended testes, varicocele, having a history of testosterone therapy, mumps, retrograde ejaculation, cryptorchidism, chromosomal abnormalities, and Y chromosome microdeletions) were excluded from the study. After retrieval, testicular tissues were suspended in the RNALater ${ }^{\circ}$ solution (Behnogen, Iran) at $4{ }^{\circ} \mathrm{C}$ overnight, and then were stored at $-80{ }^{\circ} \mathrm{C}$ until further process.

\section{RNA Extraction}

The total RNA isolation was performed using the RiboEx reagent (GeneAll, Korea) according to the manufacturer's protocol. Next, RNA was eluted in $30 \mu \mathrm{L}$ of nuclease-free water. Finally, the quantity and quality of extracted RNA were measured by a NanoDrop ${ }^{\text {tw }} 1000$ spectrophotometer and denaturing agarose gel electrophoresis.

\section{cDNA Synthesis}

To assess the expression level of hsa-mir-187-3p in testicular tissue, the first-strand complementary DNA (cDNA) was synthesized from $1 \mu \mathrm{g}$ of RNA using the stem-loop reverse transcription (RT) primer with the TaKaRa PrimeScript II 1st strand cDNA synthesis kit (Takara Bio, Ohtsu, Japan). All primers were designed based on Chen et al, ${ }^{11}$ and their details are presented in Table 1. Then, $10 \mu \mathrm{L}$ reactions were incubated in an Applied Biosystems 9700 Thermocycler for 30 minutes at $16^{\circ} \mathrm{C}$ and 30 minutes at $42^{\circ} \mathrm{C}$, followed by heat-inactivation for 5 seconds at $85^{\circ} \mathrm{C}$ and stored at $4^{\circ} \mathrm{C}$. Eventually, the obtained cDNA was checked by a spectrophotometer to evaluate its concentration.

\section{Real-Time Quantitative-RT Polymerase Chain Reaction} Real-time quantitative-RT polymerase chain reaction (qRT-PCR) was carried out with SYBR Green (TaKaRa, Japan) on a thermal Cycler StepOnePlus RT-PCR system (Applied Biosystems, Inc.). Next, the $10 \mu \mathrm{L}$ PCR reaction mixture was prepared using the ExiLENT SYBR ${ }^{\circ}$ Green PCR Master Mix (Exiqon, Denmark) following the manufacturer's protocol. The quantitative PCR condition included the initial denaturation at $95^{\circ} \mathrm{C}$ for 10 minutes and 40 cycles consisting of a denaturation step at $95^{\circ} \mathrm{C}$ for 15 seconds, followed by an annealing step at $60^{\circ} \mathrm{C}$ for 30 seconds, and finally, an extension step at $72^{\circ} \mathrm{C}$ for 30 seconds. To ensure product uniformity, a melting curve was made by increasing the temperature from $65^{\circ} \mathrm{C}$ to $95^{\circ} \mathrm{C}$ at the end of every run. Then, PCR was conducted in triplicate, and all reverse transcriptase reactions (i.e., RT minus controls and non-template controls) were run in duplicate. In addition, the average cycle of threshold $(\mathrm{Ct})$ values was computed for further analysis. Using the $\Delta \Delta \mathrm{Ct}$ method, data were analyzed and the U6 snRNA expression level was used as the endogenous reference.

Table 1. Primer Sequences for hsa-miR-187-3p and U6

\begin{tabular}{lcl}
\hline Gene & Primer & Sequence \\
\hline Hsa-miR-187-5p (MIMAT0000262) & RT & 5'- GTCGTATCCAGTGCAGGGTCCGAGGTATTCGCACTGGATACGACCCGGCT-3' \\
& $\mathrm{F}$ & 5'-AAGCGTCGTGTCTTGTGTTG-3' $^{\prime}$ \\
U6 & RT & $5^{\prime}$ - GTCGTATCCAGTGCAGGGTCCGAGGTATTCGCACTGGATACGACAATATG -3' \\
(Gene ID: 26827) & $\mathrm{F}$ & $5^{\prime}$-ATGACGCAAATTCGTGAAGC-3' \\
& $\mathrm{R}$ & 5'-CAGTGCAGGGTCCGAGGTA-3' $^{\prime}$
\end{tabular}

Note: RT: Reverse transcription; F: Forward; R: Reverse. 


\section{Statistical Analyses}

The statistical analyses of this study were conducted using SPSS, version 18.0.1 (SPSS Inc., Chicago, IL), and the $P$ value less than 0.05 was considered statistically significant. Finally, the independent t-test was utilized to compare the mean expression level of the candidate miRNA between NOA and OA groups.

\section{Results}

Infertile men participating in this study were classified as NOA (SCOS) and OA based on histological analysis. As shown in Figure 1, the relative expression level of testicular hsa-miR-187 significantly increased by 10.57 fold $(P=0.04)$ in individuals with idiopathic SCOS compared with OA men.

\section{Discussion}

The current study assessed the expression level of testicular hsa-miR-187 in idiopathic NOA patients and OA infertile men by qRT-PCR. The hsa-miR-187 expression was significantly up-regulated in the testis tissue of the NOA group compared with OA men. It should be noted that miR-187 is speculated to have a role in the regulation of proliferation and apoptosis in various cells. Furthermore, it is strongly suggested that miR-187 reduces the ability of cancer cells to survive, inhibits their growth, and induces their apoptosis. ${ }^{12}$ One hypothesis is that miR-187 induces apoptosis by down-regulating $\mathrm{Bcl}$-2. As an anti-apoptotic molecule, $B c l-2$ is expressed in disparate cell types although it is not clear whether it acts as a target regulated by miR-187. In their study, He et al showed that miR-187 decreases the expression level of $\mathrm{Bcl}-2$ in SiHa cells. They

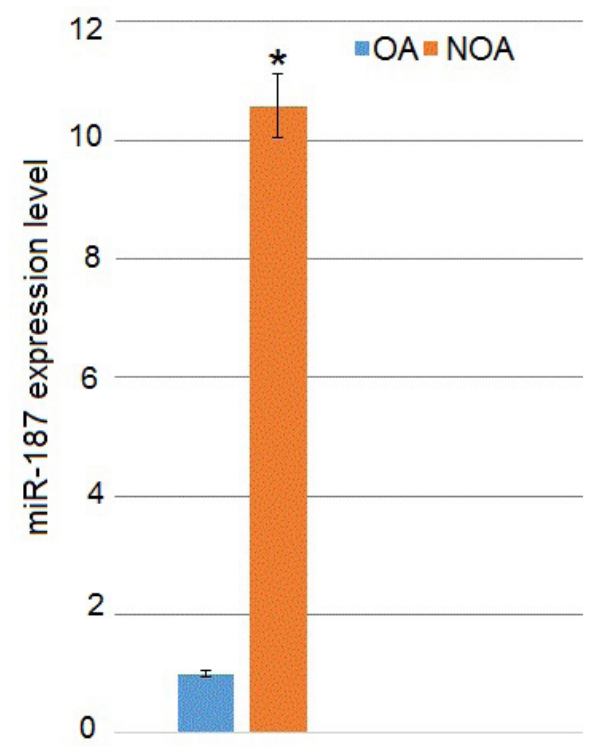

Figure 1 .The miR-187 Expression in Infertile Men With NOA Compared With OA Patients

Note. NOA: Non-obstructive azoospermia; OA: Obstructive azoospermia; The expression level of testicular miR-187 meaningfully increased $(P<0.05)$ in NOA men compared with OA men $\left({ }^{*} P<0.05\right)$. further found that apoptosis in SiHa cells is augmented as a result of $B c l-2$ inhibition induced by miR- 187 so that the upregulation of $\mathrm{Bcl}-2$ deducts the pro-apoptotic effect of miR-187. ${ }^{13}$ To the best of our knowledge, the present study first reported that hsa-miR-187 is significantly linked to NOA. However, the underlying pathway through which miR-187 contributes to normal spermatogenesis needs further investigation. According to previous evidence, miRNAs modulate diverse cellular phenomena such as growth and proliferation. ${ }^{14}$ Moreover, spermatogenesis is among the most organized and complicated processes of cellular differentiation. Spermatogonial proliferation, as the first step of spermatogenesis, helps the stem cells to maintain and eventuate the cycle of the spermatogenetic leading to the formation of spermatozoa. ${ }^{15}$ Additionally, the differentiation of spermatogonia to spermatozoa is the second step of spermatogenesis which happens through a meiotic phase and sequent spermiogenesis. Similarly, apoptosis occurs during normal spermatogenesis in testis germ cells. ${ }^{16}$ In patients with different severity rates of spermatogenic disorders, apoptosis has been proved to be a frequent event in germ cells. ${ }^{17,18}$ For example, Lin et al approved that increased apoptosis increased in infertile men with maturation arrest and hypospermatogenesis. ${ }^{19}$ In other studies, transgenic mice overexpressing $\mathrm{Bcl}-2$ or underexpressing Bax developed seminiferous tubule impairments with the cumulation of atypical premeiotic germ cells without mature spermatozoa, resulting in male infertility. ${ }^{20,21}$ Likewise, Amir et al reported that the altered expressions of pro-apoptotic Bax and voltage-dependent anion channel 1 , and anti-apoptotic $B c l-2$ are linked to oligozoospermia. ${ }^{22}$ However, the molecular mechanism underlying apoptosis in spermatogenesis has not been completely elucidated and requires further analysis.

\section{Conclusion}

In general, our findings indicated that testicular hsamiR-187 is significantly up-regulated in NOA patients with complete spermatogenic failure. Accordingly, underlying pathways regarding the link between miR-187 and spermatogenesis need thorough evaluations.

\section{Ethical Approval}

This study was originally approved by the Ethics Committee of Tarbiat Modares University, Tehran, Iran (IR.TMU.REC.1395.382).

\section{Conflict of Interest Disclosure \\ The authors declare there is no conflict of interests.}

\section{Authors' Contribution}

FP carried out the laboratory experiments and wrote the manuscript. In addition, HMsupervised and designed the scientific work and then edited the manuscript. Further, MASG selected the patients and confirmed the clinical diagnosis. Eventually, ME analyzed the results. All authors 
read and authorized the final manuscript.

\section{Acknowledgments}

The present article is part of a Ph.D. thesis financially supported by the Research Department of the School of Medical Sciences, Tarbiat Modares University, Tehran, Iran.

\section{References}

1. Hamada A, Esteves SC, Nizza M, Agarwal A. Unexplained male infertility: diagnosis and management. Int Braz J Urol. 2012;38(5):576-594. doi:10.1590/s167755382012000500002

2. Hellani A, Al-Hassan S, Iqbal MA, Coskun S. Y chromosome microdeletions in infertile men with idiopathic oligo- or azoospermia. J Exp Clin Assist Reprod. 2006;3:1. doi:10.1186/1743-1050-3-1

3. Chen K, Rajewsky N. The evolution of gene regulation by transcription factors and microRNAs. Nat Rev Genet. 2007;8(2):93-103. doi:10.1038/nrg1990

4. Friedman RC, Farh KK, Burge CB, Bartel DP. Most mammalian mRNAs are conserved targets of microRNAs. Genome Res. 2009;19(1):92-105. doi:10.1101/ gr.082701.108

5. He Z, Kokkinaki M, Pant D, Gallicano GI, Dym M. Small RNA molecules in the regulation of spermatogenesis. Reproduction. 2009;137(6):901-911. doi:10.1530/rep-080494

6. Wang C, Yang C, Chen X, et al. Altered profile of seminal plasma microRNAs in the molecular diagnosis of male infertility. Clin Chem. 2011;57(12):1722-1731. doi:10.1373/ clinchem.2011.169714

7. Liu T, Cheng W, Gao Y, Wang H, Liu Z. Microarray analysis of microRNA expression patterns in the semen of infertile men with semen abnormalities. Mol Med Rep. 2012;6(3):535-542. doi:10.3892/mmr.2012.967

8. Wu W, Hu Z, Qin Y, et al. Seminal plasma microRNAs: potential biomarkers for spermatogenesis status. Mol Hum Reprod. 2012;18(10):489-497. doi:10.1093/molehr/gas022

9. Dou C, Liu Z, Xu M, et al. miR-187-3p inhibits the metastasis and epithelial-mesenchymal transition of hepatocellular carcinoma by targeting S100A4. Cancer Lett. 2016;381(2):380-390. doi:10.1016/j.canlet.2016.08.011

10. Cooper TG, Noonan E, von Eckardstein S, et al. World Health Organization reference values for human semen characteristics. Hum Reprod Update. 2010;16(3):231-245. doi:10.1093/humupd/dmp048

11. Chen C, Ridzon DA, Broomer AJ, et al. Real-time quantification of microRNAs by stem-loop RT-PCR. Nucleic Acids Res. 2005;33(20):e179. doi:10.1093/nar/ gni178

12. Zhao J, Lei T, Xu C, et al. MicroRNA-187, down-regulated in clear cell renal cell carcinoma and associated with lower survival, inhibits cell growth and migration though targeting B7-H3. Biochem Biophys Res Commun. 2013;438(2):439-444. doi:10.1016/j.bbrc.2013.07.095

13. He CY, Yang J. miR-187 induces apoptosis of SiHa cervical carcinoma cells by downregulating Bcl-2. Genet Mol Res. 2017;16(1). doi:10.4238/gmr16018969

14. Cai Y, Yu X, Hu S, Yu J. A brief review on the mechanisms of miRNA regulation. Genomics Proteomics Bioinformatics. 2009;7(4):147-154. doi:10.1016/s1672-0229(08)60044-3

15. Phillips BT, Gassei K, Orwig KE. Spermatogonial stem cell regulation and spermatogenesis. Philos Trans R Soc Lond B Biol Sci. 2010;365(1546):1663-1678. doi:10.1098/ rstb.2010.0026

16. Blanco-Rodríguez J, Martínez-García C. Spontaneous germ cell death in the testis of the adult rat takes the form of apoptosis: re-evaluation of cell types that exhibit the ability to die during spermatogenesis. Cell Prolif. 1996;29(1):1331. doi:10.1111/j.1365-2184.1996.tb00091.x

17. Print CG, Loveland KL. Germ cell suicide: new insights into apoptosis during spermatogenesis. Bioessays. 2000;22(5):423-430. doi:10.1002/(sici)15211878(200005)22:5<423::aid-bies4>3.0.co;2-0

18. Tesarik J, Greco E, Cohen-Bacrie P, Mendoza C. Germ cell apoptosis in men with complete and incomplete spermiogenesis failure. Mol Hum Reprod. 1998;4(8):757762. doi:10.1093/molehr/4.8.757

19. Lin WW, Lamb DJ, Wheeler TM, Lipshultz LI, Kim ED. In situ end-labeling of human testicular tissue demonstrates increased apoptosis in conditions of abnormal spermatogenesis. Fertil Steril. 1997;68(6):1065-1069. doi:10.1016/s0015-0282(97)00372-5

20. Furuchi T, Masuko K, Nishimune Y, Obinata M, Matsui Y. Inhibition of testicular germ cell apoptosis and differentiation in mice misexpressing $\mathrm{Bcl}-2$ in spermatogonia. Development. 1996;122(6):1703-1709.

21. Knudson CM, Tung KS, Tourtellotte WG, Brown GA, Korsmeyer SJ. Bax-deficient mice with lymphoid hyperplasia and male germ cell death. Science. 1995;270(5233):96-99. doi:10.1126/science.270.5233.96

22. Amir A, Yanwirasti, Asmarinah, Oenzil F. Alteration Expression of Bax, Bcl-2 and VDAC1 Genes in Oligozoospermic and Fertile Subjects. Pak J Biol Sci. 2016;19(2):71-76. doi:10.3923/pjbs.2016.71.76 